\title{
Dynamics of periodic multi-component Bose-Einstein condensates
}

\author{
Bernard Deconinck* ${ }^{*}$ J. Nathan Kutz ${ }^{\dagger}$, Matthew S. Patterson ${ }^{\dagger}$, and Brandon W. Warner ${ }^{\dagger}$ \\ *Department of Mathematics, Colorado State University, Fort Collins, CO 80523, USA \\ ${ }^{\dagger}$ Department of Applied Mathematics, University of Washington, Seattle, WA, 98195 \\ Short Title: Dynamics of periodic multicomponent BECs \\ PACS numbers: 03.75.Lm, 03.75.Mn, 02.30.Jr, 02.60.Cb, 02.70.Hm
}

January 24, 2003

\begin{abstract}
The cubic vector nonlinear Schrödinger equation with an external trigonometric potential models a quasi-dimensional multi-component Bose-Einstein condensate trapped in a standinglight wave. We construct families of exact stationary solutions for the more general case of an elliptic function potential. Some of these solutions degenerate to zero as the effect of the external potential disappears, whereas others limit to solutions of the free vector nonlinear Schrödinger equation. The stability of these solutions is examined both analytically and numerically. The stability results depend on the nature of the atomic interactions both within the components but also between components. As in the scalar case (one component) with repulsive interaction, all linearly stable solutions are deformations of the ground state of the linear Schrödinger equation. Unlike the scalar case with attractive interaction, no solutions are found to be stable if there is any attractive interaction present.
\end{abstract}

\section{Introduction}

The experimental realization of Bose-Einstein condensation [1] provides one of a few examples of a macroscopic quantum phenomenon $[2,3]$. The creation of a Bose-Einstein condensate (BEC) is accomplished experimentally by super-cooling certain dilute alkali gases to below a critical temperature, generally in the microKelvin range (see [4] for an overview). Currently, the entire experiment takes place in an electromagnetic trap [5] on the order of millimeters or smaller. In most experiments, the electromagnetic trap results in a confining potential which is harmonic, or very nearly so. In addition to confining potentials, recent interest has focused on sinusoidal potentials, obtained by placing the BEC in a standing light wave [6]. Possible applications of BECs in sinusoidal potentials include the study of phase coherence [7, 8, 9], matter-wave diffraction [10], matter-wave gratings and matter-wave transport [11], and quantum logic [12, 13].

In most experiments, only one atomic species is involved, and only one quantum state of this atomic species participates in the condensation. However, experiments where two distinct BECs are present in the same trap are possible: in $[14,15]$ two different condensates correspond to different 
spin states of ${ }^{87} \mathrm{Rb}$; in [16] an optical trap was used for the confinement of a ${ }^{23} \mathrm{Na}$ spinor condensate, effectively resulting in distinct interacting condensates. More recently, interacting condensates of different atomic species were produced [17].

In this paper, the mean-field dynamics and stability of quasi-one-dimensional, coupled, dilutegas BECs in a sinusoidal potential are examined. The case of a single condensate in such a potential was already considered in $[18,19]$ (BEC with repulsive interatomic interaction) and [20] (BEC with attractive interatomic interaction).

The mean-field theory for the ground state of a single macroscopic BEC wave function is constructed using the Hartree-Fock approximation [21], assuming a full occupancy rate for the condensate (i.e. zero temperature), and a hard interaction between atoms. The resulting governing equation is the Gross-Pitaevskii equations $[22,23]$. Note that the asymptotic exactness of this equation was demonstrated in $[24,25]$. The number of spatial dimensions that are considered is a crucial factor for the dynamics of the condensate. One-dimensional, two-dimensional and threedimensional BECs all behave in radically different manners $[26,27]$. The quasi-one-dimensional regime is relevant when the transverse dimensions of the condensate are on the order of its healing length, and both are much smaller than its longitudinal dimension [28, 29]. Thus the condensate has the form of an ellipsoid stretched along one of its major axes. In this regime the BEC remains phase coherent and the governing equation is one-dimensional: the Gross-Pitaevskii equation reduces to a one-dimensional nonlinear Schrödinger equation (NLS) with an external potential. The quasione-dimensional regime should be contrasted with a truly one-dimensional theory which requires transverse dimensions on the order of or less than the atomic interaction length.

If more than one distinct condensate is trapped in the confining potential the Gross-Pitaevskii equation is no longer sufficient. In this case, the wave function for each BEC satisfies its own GrossPitaevskii equation, coupled to the others by nonlinear mean-field interactions (see, for instance $[4,31,30])$. Thus, in the quasi-one-dimensional regime, the dynamics is governed by a set of coupled NLS equations with an external potential. This dynamics depends heavily on the sign of the coupling constants, which characterize the strength of the interaction between the different condensates. Myatt et al. [14] observed experimentally that the BECs corresponding to different spin states of ${ }^{87} \mathrm{Rb}$ have a repulsive interaction, implying a positive coupling constant. However, for other atomic species and their isotopes, the coupling constant can be negative, resulting in various possibilities: BECs with repulsive interatomic interaction and repulsive or attractive coupling, BECs with attractive interatomic interaction and repulsive or attractive coupling, and mixes of these two scenarios. All of these are considered for two classes of exact solutions of the coupled NLS equations with a sinusoidal potential and generalizations thereof. Note that the stability of such coupled condensates was considered previously in $[4,31]$, for the case of harmonic confinement, using a variational reduction.

The paper is outlined as follows. In the next section we discuss the governing evolution equations along with the confining potential. Section 3 introduces two classes of exact solutions of these equations, which are valid for an arbitrary number of interacting condensates. Partial stability results for these exact solutions are given in Section 4. These stability results are confirmed and augmented by numerical simulations in Section 5. A brief discussion of the results and their significance in an experimental context concludes the paper. 


\section{Governing equations}

The governing equations for the interaction of $n \geq 2$ condensates are [4]

$$
i \hbar \frac{\partial \Psi_{j}}{\partial t}=-\frac{\hbar^{2}}{2 m_{j}} \frac{\partial^{2} \Psi_{j}}{\partial x^{2}}+V_{j}(x) \Psi_{j}+\sum_{l=1}^{n} \frac{2 \pi \hbar^{2} a_{j l}}{m_{j l}}\left|\Psi_{l}\right|^{2} \Psi_{j}, \quad j=1, \ldots, n .
$$

Here, $\Psi_{j}(x, t)$ is the wave function for the $j$-th condensate; $V_{j}(x)$ is the external potential experienced by the $j$-th condensate. In most situations $V_{j}(x)=V(x)$ is identical for all $j=1, \ldots, n$. However, because of the different properties of constituting atoms of the different condensates, it is possible for different condensates in the same physical trap to experience different external potentials. Also, $m_{j}$ is the atom mass of the atom species of the $j$-th condensate; $m_{j l}=m_{j} m_{l} /\left(m_{j}+m_{l}\right)$ is the reduced mass corresponding to atom species of the $j$-th and $l$-th condensate; finally, $a_{j l}=a_{l j}$ is the $s$-wave scattering length between the atomic species $j$ and $l$. The sign of this $s$-wave scattering length determines the nature of the interaction between the different atomic species: a positive value gives rise to a repulsive interatomic interaction, whereas a negative value causes an attractive interaction.

The equations (1) can be made nondimensional by rescaling of the dependent and independent variables. After this rescaling, the equations have the form

$$
i \frac{\partial \Psi_{j}}{\partial t}=-\frac{1}{2 \mu_{j}} \frac{\partial^{2} \Psi_{j}}{\partial x^{2}}+V_{j}(x) \Psi_{j}+\sum_{l=1}^{n} \alpha_{j l}\left|\Psi_{l}\right|^{2} \Psi_{j}, \quad j=1, \ldots, n .
$$

We have not introduced new symbols for the dimensionless quantities, so as not to overburden the notation. The symmetric matrix $\boldsymbol{\alpha}=\left(\alpha_{j k}\right)_{j, k=1}^{n}$ contains all information about the nature of the interatomic interactions. Its entries are referred to as interaction coefficients. The parameters $\mu_{j}, j=1, \ldots, n$, play the role of effective masses. Current experiments $[14,15,16]$ with multiple condensates use different spin states or distinct isotopes of one atomic species, so that all $\mu_{j}$, $j=1, \ldots, n$, are equal. In that case, the parameters $\mu_{j}, j=1, \ldots, n$, can be removed by another scaling transformation, so that effectively $\mu_{j}=1, j=1, \ldots, n$. This is the case used in the numerical simulations of Section 5.

The external potentials considered in this paper are generalizations of the sinusoidal, standing light wave potential [6]:

$$
V_{j}(x)=-V_{0 j} \operatorname{sn}^{2}(m x, k),
$$

with $-V_{0 j}$ an amplitude parameter. The function $\operatorname{sn}(m x, k)$ denotes Jacobi's elliptic sine function [32]. As the elliptic modulus $k \rightarrow 0, \operatorname{sn}(m x, k) \rightarrow \sin (m x)$. The Jacobi sine function $\operatorname{sn}(m x, k)$ is periodic in $x$ for every value of $k \in[0,1)$, with period given by the elliptic integral $4 K(k) / m=$ $(4 / m) \int_{0}^{\pi / 2} d z / \sqrt{1-k^{2} \sin ^{2} z}$. Note that this period approaches infinity as $k \rightarrow 1$. Thus, as $k \rightarrow 1$, the potential (3) is a periodic lattice of separated peaks or troughs, depending on the sign of $V_{0 j}$. Hence, by changing the parameter $k$, various interesting regimes of the BECs are considered. This is the reason for considering potentials that are more general than the standing light wave potential. From (3) it follows that the potentials considered here are identical for all condensates, modulo an amplitude factor. The - sign is part of the definition of the potential because in the important limit, as the elliptic modulus $k \rightarrow 0, V_{j}(x)=-V_{0 j} \sin ^{2}(m x)$, which equals $V_{0 j} \cos 2 m x$, up to an additive constant. 


\section{Exact solutions}

This section gives a dictionary of the families of exact solutions we were able to find. These families are extensions of the families found for the scalar case, discussed in [19, 20]. There are two distinct types of solutions. Both types of solutions require all components of the condensate to have the same functional form, i.e., the types cannot be mixed.

\section{Type A}

Solutions of Type A are solutions for which the density of each condensate $\left|\Psi_{k}(x, t)\right|^{2}$ is a quadratic function of $\operatorname{sn}(x, k)$. These solutions are given by $\Psi_{j}(x, t)=\exp \left(-i \omega_{j} t+i \theta_{j}(x)\right) r_{j}(x), \theta_{j x}=$ $c_{j} / r_{j}^{2}(x), j=1, \ldots, n$, with

$$
\begin{aligned}
r_{j}^{2} & =A_{j} \mathrm{sn}^{2}(m x, k)+B_{j}, \\
\sum_{l=1}^{n} \alpha_{j l} A_{l} & =V_{0 j}+m^{2} k^{2} \mu_{j}, \\
\omega_{j} & =\frac{1}{2} m^{2}\left(1+k^{2}\right) \mu_{j}+\frac{1}{2} m^{2} k^{2} \mu_{j} \frac{B_{j}}{A_{j}}+\sum_{l=1}^{n} \alpha_{j l} B_{l}, \\
c_{j}^{2} & =m^{2} \frac{B_{j}}{A_{j}}\left(A_{j}+B_{j}\right)\left(A_{j}+k^{2} B_{j}\right),
\end{aligned}
$$

and $j=1, \ldots, n$. Thus, in order to uniquely determine the amplitude of the elliptic oscillations $A_{j}, j=1, \ldots, n$, the matrix $\boldsymbol{\alpha}$ needs to be inverted. The resulting family of solutions has $n$ free parameters $B_{j}, j=1, \ldots, n$, playing the role of an offset. Trivial-phase solutions $\left(\theta_{j}(x)=0\right)$ occur for those values of $B_{j}$ for which the corresponding $c_{j}=0$. Specifically,

$$
\begin{aligned}
B_{j}=0: & \Psi_{j}(x, t)=\sqrt{A_{j}} \operatorname{sn}(m x, k) e^{-i \omega_{j} t} \\
B_{j}=-A_{j}: & \Psi_{j}(x, t)=\sqrt{-A_{j}} \operatorname{cn}(m x, k) e^{-i \omega_{j} t} \\
B_{j}=-A_{j} / k^{2}: & \Psi_{j}(x, t)=\sqrt{-A_{j} / k^{2}} \operatorname{dn}(m x, k) e^{-i \omega_{j} t}
\end{aligned}
$$

Here $\operatorname{cn}(m x, k)$ and $\operatorname{dn}(m x, k)$ denote the Jacobian elliptic cosine and the third Jacobian elliptic function respectively [32]. Note that it is possible for some component solutions $\Psi_{j}(x, t)$ to have trivial phase, whereas others may have nontrivial phase. The solutions (4a-d) have both trigonometric $(k \rightarrow 0, \mathrm{sn} \rightarrow \sin )$ and hyperbolic $(k \rightarrow 1, \mathrm{sn} \rightarrow$ tanh $)$ limits. As mentioned in the previous sections, especially the trigonometric limit is relevant for applications. These limiting solutions are discussed in more detail in [19]. In contrast to the trivial-phase limit, it is not possible for some components to have trigonometric or hyperbolic profiles, while others have elliptic profiles, since the elliptic parameter $k$ is identical for all components.

In order to use the trivial-phase solutions in numerical simulations, it is necessary to establish their existence regions, i.e, the regions in parameter space where a certain type of solution is defined. This is a daunting task for $n \geq 3$, and is very tedious even for $n=2$. It is essential to 
reduce the dimension of parameter space as much as possible. The parameters in question are the $n(n+1) / 2$ entries of the matrix $\boldsymbol{\alpha}$, the $n$ reduced masses $\mu_{j}, j=1, \ldots, n$, and the $n$ components of the potential amplitude $V_{0 j}, j=1, \ldots, n$. In what follows, the effective masses $\mu_{j}, j=1, \ldots, n$ are all equated to one. Although this does affect the size and shape of the distinct existence regions, it does not affect their number, since all the effective masses are positive. Another observation is that by the use of a scaling transformation, the diagonal elements of $\boldsymbol{\alpha}$ can be rescaled to \pm 1 .

As an example, consider the case of $n=2$, with both species having repulsive self-interaction: $\alpha_{11}=\alpha_{22}=1$. Then the equations determining $A_{1}$ and $A_{2}$ are

$$
\left\{\begin{array}{l}
A_{1}+\alpha A_{2}=V_{01}+m^{2} k^{2}, \\
\alpha A_{1}+A_{2}=V_{02}+m^{2} k^{2},
\end{array}\right.
$$

with $\alpha=\alpha_{12}=\alpha_{21}$. From (5a-c) it follows that the solution (5a) is defined in regions where the corresponding amplitude is positive, whereas the solutions (5b-c) are defined in regions where the corresponding amplitude is negative. This is illustrated in Figure 1a for $\alpha>1$. Other regimes exist for $\alpha<1$, and for different choices of the self-interaction.

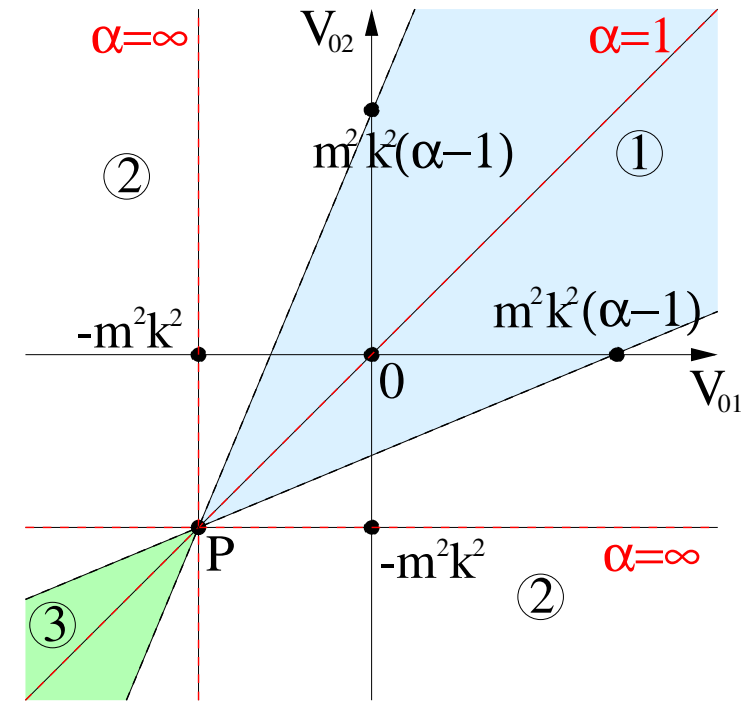

(a)

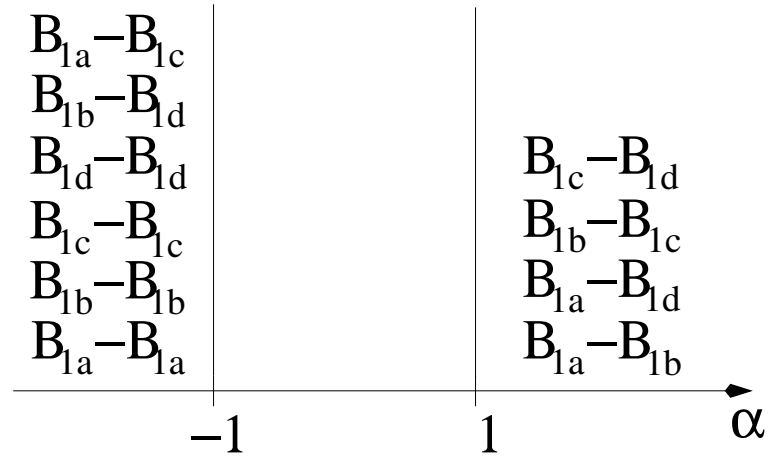

(b)

Figure 1: Parameter space for a two-component condensate with repulsive self-interactions. (a) Parameter space for Type A solutions with coupling interaction $\alpha>1$. In Region 1, only the trivial-phase sn $-\mathrm{sn}$ solution is defined. In Region 2, trivial-phase solutions $\mathrm{sn}-\mathrm{cn}$ and $\mathrm{sn}-\mathrm{dn}$ are possible, whereas in Region 3, the trivial-phase solution types $\mathrm{cn}-\mathrm{cn}$, cn $-\mathrm{dn}$ and $\mathrm{dn}-\mathrm{dn}$ exist. (b) Parameter space for Type $\mathrm{B}_{1}$.

\section{Type B}

Solutions of Type B are solutions for which the density of each condensate $\left|\Psi_{k}(x, t)\right|^{2}$ is a linear function of a Jacobian elliptic function. For these solutions, the potential strengths are limited by 


$$
V_{0 j}=-\frac{3}{8} m^{2} k^{2} \mu_{j}, \quad j=1, \ldots, n .
$$

It is clear from this that these solutions have no trigonometric limit: (2) degenerates to the VNLS equation without external potential, which does not have solutions whose density is linear in terms of Jacobi elliptic functions. Indeed, all solutions of Type B approach zero in the trigonometric limit. Hyperbolic limits do exist, however. All of the solution families of Type $B$ have $n$ free parameters. It is convenient to choose these as the amplitude of the oscillations $A_{j}, j=1, \ldots, n$. As before the expressions for these solutions use an amplitude-phase decomposition: $\Psi_{j}(x, t)=$ $r_{j}(x) \exp \left(-i \omega_{j} t+i \theta_{j}(x)\right), \theta_{j x}=c_{j} / r_{j}^{2}(x), j=1, \ldots, n$. Then

- Type $\mathbf{B}_{1}$ :

$$
\begin{aligned}
r_{j}^{2} & =A_{j} \operatorname{sn}(m x, k)+B_{j}, \\
B_{j} & =\frac{-4 A_{j}}{\mu_{j} m^{2} k^{2}} \sum_{l=1}^{n} \alpha_{j l} A_{l}, \\
\omega_{j} & =\frac{1}{8} \mu_{j} m^{2}\left(1+k^{2}\right)-\frac{1}{8} \mu_{j} m^{2} k^{2} \frac{B_{j}^{2}}{A_{j}^{2}}+\sum_{l=1}^{n} \alpha_{j l} B_{l}, \\
c_{j}^{2} & =\frac{m^{2}}{4 A_{j}^{2}}\left(B_{j}^{2}-A_{j}^{2}\right)\left(A_{j}^{2}-k^{2} B_{j}^{2}\right) .
\end{aligned}
$$

Several trivial-phase solutions exist, for those values of $A_{j}$ that annihilate $c_{j}^{2}$ : $\Psi_{j}(x, t)=$ $\sigma_{j} \sqrt{A_{j}(\operatorname{sn}(m x, k)+1)} \exp \left(-i \omega_{j} t\right)\left(A_{j}=B_{j}>0\right.$, Type $\left.\mathrm{B}_{1 a}\right) ; \Psi_{j}(x, t)=\sigma_{j} \sqrt{A_{j}(\operatorname{sn}(m x, k)-1)}$ $\exp \left(-i \omega_{j} t\right)\left(A_{j}=-B_{j}<0\right.$, Type $\left.\mathrm{B}_{1 b}\right) ; \Psi_{j}(x, t)=\sqrt{A_{j}(\operatorname{sn}(m x, k)+1 / k)} \exp \left(-i \omega_{j} t\right)\left(A_{j}=\right.$ $k B_{j}>0$, Type $\left.\mathrm{B}_{1 c}\right)$; and $\Psi_{j}(x, t)=\sqrt{A_{j}(\operatorname{sn}(m x, k)-1 / k)} \exp \left(-i \omega_{j} t\right)\left(A_{j}=-k B_{j}<0\right.$, Type $\left.\mathrm{B}_{1 d}\right)$; Here $\sigma_{j}= \pm 1$, chosen so as to ensure $\Psi_{j}(x, t)$ is analytic in $x$.

- Type $\mathrm{B}_{2}$ :

$$
\begin{aligned}
r_{j}^{2} & =A_{j} \operatorname{cn}(m x, k)+B_{j}, \\
B_{j} & =\frac{4 A_{j}}{\mu_{j} m^{2} k^{2}} \sum_{l=1}^{n} \alpha_{j l} A_{l}, \\
\omega_{j} & =\frac{1}{8} \mu_{j} m^{2}\left(1+k^{2}\right)+\frac{1}{8} \mu_{j} m^{2} k^{2} \frac{B_{j}^{2}}{A_{j}^{2}}+\sum_{l=1}^{n} \alpha_{j l} B_{l}, \\
c_{j}^{2} & =\frac{m^{2}}{4 A_{j}^{2}}\left(B_{j}^{2}-A_{j}^{2}\right)\left(A_{j}^{2}+k^{2} B_{j}^{2}-k^{2} A_{j}^{2}\right) .
\end{aligned}
$$

For Type $\mathrm{B}_{2}$, two types of trivial-phase solutions exist: $\Psi_{j}(x, t)=\sigma_{j} \sqrt{A_{j}(\operatorname{cn}(m x, k)+1)}$ $\exp \left(-i \omega_{j} t\right),\left(A_{j}=B_{j}>0\right.$, Type $\left.\mathrm{B}_{2 a}\right) ;$ and $\Psi_{j}(x, t)=\sigma_{j} \sqrt{A_{j}(\operatorname{cn}(m x, k)-1)} \exp \left(-i \omega_{j} t\right)$, $\left(A_{j}=B_{j}<0\right.$, Type $\left.\mathrm{B}_{2 b}\right)$, where $\sigma_{j}$ is as before. 
- Type $\mathbf{B}_{3}$ :

$$
\begin{aligned}
r_{j}^{2} & =A_{j} \operatorname{dn}(m x, k)+B_{j}, \\
B_{j} & =\frac{4 A_{j}}{\mu_{j} m^{2}} \sum_{l=1}^{n} \alpha_{j l} A_{l}, \\
\omega_{j} & =\frac{1}{8} \mu_{j} m^{2}\left(1+k^{2}\right)+\frac{1}{8} \mu_{j} m^{2} \frac{B_{j}^{2}}{A_{j}^{2}}+\sum_{l=1}^{n} \alpha_{j l} B_{l}, \\
c_{j}^{2} & =\frac{m^{2}}{4 A_{j}^{2}}\left(A_{j}^{2}-B_{j}^{2}\right)\left(A_{j}^{2}-B_{j}^{2}-k^{2} A_{j}^{2}\right) .
\end{aligned}
$$

Here four types of trivial-phase solutions exist: $\Psi_{j}(x, t)=\sqrt{A_{j}(\operatorname{dn}(m x, k)+1)} \exp \left(-i \omega_{j} t\right)$, $\left(A_{j}=B_{j}>0\right.$, Type $\left.\mathrm{B}_{3 a}\right) ; \Psi_{j}(x, t)=\sqrt{A_{j}(\operatorname{dn}(m x, k)-1)} \exp \left(-i \omega_{j} t\right),\left(A_{j}=-B_{j}<0\right.$, Туре $\left.\mathrm{B}_{3 b}\right) ; \Psi_{j}(x, t)=\sqrt{A_{j}\left(\operatorname{dn}(m x, k)+\sqrt{1-k^{2}}\right)} \exp \left(-i \omega_{j} t\right),\left(A_{j}=B_{j} / \sqrt{1-k^{2}}>0\right.$, Type $\left.\mathrm{B}_{3 c}\right) ;$ $\Psi_{j}(x, t)=\sigma_{j} \sqrt{A_{j}\left(\operatorname{dn}(m x, k)-\sqrt{1-k^{2}}\right)} \exp \left(-i \omega_{j} t\right),\left(A_{j}=-B_{j} / \sqrt{1-k^{2}}>0\right.$, Type $\left.\mathrm{B}_{3 d}\right)$, where $\sigma_{j}$ is as before.

Note that mixing of these solution types is not allowed: it is not possible for one component of the condensate to correspond to a solution of Type $\mathrm{B}_{1}$, while another corresponds to Type $\mathrm{B}_{2}$ or $\mathrm{B}_{3}$. Mixing of different types of trivial-phase solutions of the same type is possible.

Since the potential strengths $V_{0 j}, j=1, \ldots, n$ are constrained for Type B solutions, parameter space is lower dimensional than for the solutions of Type A. Specifically, for the case of a twocomponent condensate with preset self-interactions, there is only one parameter, $\alpha$, the off-diagonal element of the interaction matrix. The $n=2$ case of Type $\mathrm{B}_{1}$ solutions with repulsive selfinteractions is illustrated in Fig. 6b. Many other regimes exist for different self-interactions and different solution types.

\section{Stability analysis}

To examine the linear stability of these exact solutions analytically, the governing equations (2) are linearized around the exact solution,

$$
\Psi_{j}(x, t)=e^{i \theta_{j}(x)-i \omega_{j} t}\left(r_{j}(x)+\epsilon \phi_{j}(x, t)\right),
$$

for a small parameter $\epsilon$. Substitution of (11) in (2) and ignoring terms containing higher than linear powers in $\epsilon$ gives

$$
\frac{\partial}{\partial t}\left(\begin{array}{c}
u \\
v
\end{array}\right)=J\left(\begin{array}{cc}
L^{+} & S \\
-S & L^{-}
\end{array}\right)\left(\begin{array}{l}
u \\
v
\end{array}\right),
$$

where $u=\left(u_{1}, \ldots, u_{n}\right)^{T}, v=\left(v_{1}, \ldots, v_{n}\right)^{T}$, and $u_{j}, v_{j}$ are the real and imaginary parts of $\phi_{j}$. Also

$$
J=\left(\begin{array}{rr}
0_{n} & 1_{n} \\
-1_{n} & 0_{n}
\end{array}\right),
$$


where $0_{n}$ and $1_{n}$ are the $n \times n$ zero and identity matrix, respectively. The $n \times n$ matrix operators $L^{+}, L^{-}, S$ are given by

$$
\begin{aligned}
\left(L^{+}\right)_{j k} & =\left\{\begin{array}{ll}
L_{j}^{+}, & j=k \\
2 \alpha_{j k} r_{j} r_{k}, & j \neq k
\end{array},\right. \\
\left(L^{-}\right)_{j k} & =\left\{\begin{array}{ll}
L_{j}^{-}, & j=k \\
0, & j \neq k
\end{array},\right. \\
(S)_{j k} & =\left\{\begin{array}{ll}
S_{j}, & j=k \\
0, & j \neq k
\end{array},\right.
\end{aligned}
$$

with

$$
\begin{array}{rlrl}
L_{j}^{+} & =-\frac{1}{2} \partial_{x}^{2}+\frac{c_{j}^{2}}{2 r_{j}^{4}}+\sum_{k=1}^{n} \alpha_{j k} r_{k}^{2}+V_{j}-\omega_{j}+2 \alpha_{j j} r_{j}^{2}, & & j=1, \ldots, n, \\
L_{j}^{-} & =-\frac{1}{2} \partial_{x}^{2}+\frac{c_{j}^{2}}{2 r_{j}^{4}}+\sum_{k=1}^{n} \alpha_{j k} r_{k}^{2}+V_{j}-\omega_{j}, & j=1, \ldots, n, \\
S_{j} & =\frac{c_{j}}{r_{j}} \partial_{x} \frac{1}{r_{j}}, & j=1, \ldots, n .
\end{array}
$$

The treatment of the linear stability analysis for nontrivial phase solutions $\left(c_{j} \neq 0\right.$, for any $j$ ) is beyond the scope of our methods, even in the scalar case $(n=1)$. However, if all condensate components are described by trivial solutions, more analysis is possible $[18,19,20]$.

In the trivial-phase case $c_{j}=0$ and thus $S_{j}=0, j=1, \ldots, n$. Upon using separation of variables $(u(x, t), v(x, t)) \rightarrow e^{\lambda t}(u(x), v(x))$ in (12), the spectral problem reduces to

$$
\left\{\begin{array}{l}
L^{-} v=\lambda u \\
L^{+} u=-\lambda v .
\end{array}\right.
$$

Thus, if the spectral problem (20) has any positive eigenvalues, then the solution is linearly unstable. However, if all eigenvalues are imaginary, then the solution is linearly stable.

Note that

$$
L^{-}\left(\begin{array}{c}
r_{1} \\
\vdots \\
r_{n}
\end{array}\right)=0,
$$

and thus $\lambda=0$ is an eigenvalue of $L^{-}$, with eigenfunction $\left(r_{1}, \ldots, r_{n}\right)^{T}$. Next, let

$$
\lambda^{ \pm}=\inf _{\|\psi\|=1}\left\langle\psi\left|L^{ \pm}\right| \psi\right\rangle
$$

where $\|\psi\|=\left(\int_{0}^{4 K(k) / m}|\psi|^{2} d x\right)^{1 / 2}$ is the standard $L^{2}$-norm, and $\left\langle\psi_{1}\left|L^{ \pm}\right| \psi_{2}\right\rangle=\int_{0}^{4 K(k) / m} \psi_{1}^{*} L^{ \pm} \psi_{2} d x$, and $\psi_{1}^{*}$ denotes the complex conjugate of $\psi_{1}$. Thus, $\lambda^{+}\left(\lambda^{-}\right)$is the smallest eigenvalue of $L^{+}\left(L^{-}\right)$. Next, $L^{+}=L^{-}+2\left(\boldsymbol{\alpha} R^{2}\right)$, where $\left(\boldsymbol{\alpha} R^{2}\right)_{j k}=\alpha_{j k} r_{j} r_{k}$, for $j, k=1, \ldots, n$.

Then 
Lemma 1 The $n \times n$ matrix $\left(\boldsymbol{\alpha} R^{2}\right)_{j, k=1}^{n}$ is positive (negative) definite if and only if the matrix $(\boldsymbol{\alpha})_{j, k=1}^{n}$ is positive (negative) definite.

Proof The matrix $\left(\boldsymbol{\alpha} R^{2}\right)_{j, k=1}^{n}$ is positive definite if and only if all its principal minors are positive [33]. The principal minors of $\left(\boldsymbol{\alpha} R^{2}\right)_{j, k=1}^{n}$ are the determinants of $\left(\boldsymbol{\alpha} R^{2}\right)_{j, k=1}^{m}$, for $m=1, \ldots, n$. But every element of the $j$-th row of the matrix in the $m$-th principal minor of $\left(\boldsymbol{\alpha} R^{2}\right)_{j, k=1}^{n}$ contains $r_{j}$, and every element of the $k$-th column of the matrix in the $m$-th principal minor contains $r_{k}$, thus

$$
\operatorname{det}\left(\boldsymbol{\alpha} R^{2}\right)_{j, k=1}^{m}=\left(\prod_{j=1}^{n} r_{j}^{2}\right) \operatorname{det}(\boldsymbol{\alpha})_{j, k=1}^{m},
$$

for $m=1, \ldots, n$. Here $\operatorname{det}(\boldsymbol{\alpha})_{j, k=1}^{m}$ is the $m$-th principal minor of $(\boldsymbol{\alpha})_{j, k=1}^{n}$, which proves the lemma. The negative definite case is identical to the proof given here, with "positive" replaced by "negative".

The above lemma lead to the following

Theorem 1 If $\boldsymbol{\alpha}$ is positive definite, then

- if $r_{j}(x)>0$ for all $x, j=1, \ldots, n$ then the trivial-phase solution $\Psi_{j}(x, t)=e^{-i \omega_{j} t} r_{j}(x)$ is linearly stable;

- if $r_{j}(x)<0$ for any $x$, for any $j \in[1, n]$ and the matrix operator $L^{+}$is positive, then the trivial-phase solution $\Psi_{j}(x, t)=e^{-i \omega_{j} t} r_{j}(x)$ is linearly unstable.

Proof If $\boldsymbol{\alpha}$ is a positive definite matrix, then by Lemma $1, L^{+}-L^{-}=2\left(\boldsymbol{\alpha} R^{2}\right)$ is a positive operator, or $L^{+}>L^{-}$. If $r_{j}(x)>0$ for all $x, j=1, \ldots, n$, then $\left(r_{1}, \ldots, r_{n}\right)^{T}$ is a ground state of $L^{-}$[34], and $\lambda^{-}=0$ or $L^{+}>L^{-}$is a positive operator. Then there exists a self-adjoint positive operator $H$,

$$
H=\left(L^{+}\right)^{1 / 2} L^{-}\left(L^{+}\right)^{1 / 2} .
$$

In terms of this operator, the spectral problem (20) is rewritten as

$$
\left(H+\lambda^{2} 1_{n}\right) w=0,
$$

with $w=\left(L^{+}\right)^{1 / 2} u$. From this representation and the fact that $H$ is positive, it follows that $\lambda^{2}<0$, thus all $\lambda$ are imaginary. This proves the first assertion.

To prove the second assertion, note that if $r_{j}(x)<0$ for any $x$, for any $j \in[1, n]$, then $\left(r_{1}, \ldots, r_{n}\right)^{T}$ is not the groundstate of $L^{-}[34]$, thus $\lambda^{-}<0$, and there exists a $\varphi(x)$ such that $\left\langle\varphi\left|L^{-}\right| \varphi\right\rangle<0$. Assuming $\lambda^{+}>0$, the construction leading to (22) remains valid. In particular, the operator $H$ in (21) is still defined. Let $\varphi_{0}=\left(L^{+}\right)^{-1 / 2} \varphi$, then

$$
\begin{aligned}
\left\langle\varphi_{0}|H| \varphi_{0}\right\rangle & =\left\langle\varphi_{0}\left|\left(L^{+}\right)^{1 / 2} L^{-}\left(L^{+}\right)^{1 / 2}\right| \varphi_{0}\right\rangle \\
& =\left\langle\varphi\left|L^{-}\right| \varphi\right\rangle \\
& <0
\end{aligned}
$$


from which it follows that positive $\lambda^{2}$ in (22) exist, leading to the presence of unstable modes. This establishes the second assertion.

This theorem provides two criteria for establishing linear stability analytically for positive definite $\boldsymbol{\alpha}$, i.e., the case of overall repulsive interaction. The first of these criteria is very practical, requiring no more than an inspection of the solution. The second criterion is less practical, requiring proving that $L^{+}$is a positive operator. If $\boldsymbol{\alpha}$ is negative definite, (overall attractive interaction) the following negative result holds:

Theorem 2 If $\boldsymbol{\alpha}$ is negative definite, and $r_{j}(x)>0$ for all $x, j=1, \ldots, n$, then the trivial-phase solution $\Psi_{j}(x, t)=e^{-i \omega_{j} t} r_{j}(x)$ is linearly unstable.

Proof If $r_{j}(x)>0$ for all $x, j=1, \ldots, n$, then $\left(r_{1}, \ldots, r_{n}\right)^{T}$ is a ground state of $L^{-}$[34], and $\lambda^{-}=0$ or $L^{-}$is a non-negative operator. Then there exists a self-adjoint positive operator $H$,

$$
H=\left(L^{-}\right)^{1 / 2} L^{+}\left(L^{-}\right)^{1 / 2}
$$

In terms of this operator, the spectral problem (20) is rewritten as $(22)$, with $w=\left(L^{-}\right)^{1 / 2} u$. If $\boldsymbol{\alpha}$ is a negative definite matrix, then by Lemma $1, L^{-}-L^{+}=-2\left(\boldsymbol{\alpha} R^{2}\right)$ is a positive operator, or $L^{-}>L^{+}$, hence $\lambda^{+}<\lambda^{-}$. Using a similar argument as to prove the second assertion of Theorem 1 concludes the proof.

Theorems 1 and 2 cover only few of the exact solutions described in the previous section. No information is obtained about the stability of nontrivial phase solutions, or about the case when $\boldsymbol{\alpha}$ is neither positive or negative definite. For all of these cases, we resort to numerical methods.

\section{$5 \quad$ Numerical simulations}

In this section, the results of the numerical simulations are discussed. Equation (2) is solved numerically with initial conditions chosen from the set of exact solutions given in Section 3. The numerical procedure uses a fourth-order Runge-Kutta method to advance in time and a filtered pseudo-spectral method in space. For each numerical experiment a small amount of white noise is added as a perturbation to the initial condition.

The figures in this section show the dynamics, as obtained from the numerical computations, for the $n$ interacting condensates in side-by-side stacks. Each stack displays, from top to bottom, the modulus of the solution squared $\left|\Psi_{j}(x, t)\right|^{2}$, a contour plot of the same, the potential $V_{j}(x)=V(x)$, and the inverse tangent of the Fourier spectrum of $\left|\Psi_{j}(x, t)\right|$, $\arctan \left|\tilde{\Psi}_{j}(k)\right|$. For convenience, we use "repulsive" to imply that all $n$ condensates are self-repelling, whereas "attractive" is used to denote that all $n$ condensates are self-attracting. The connotation "mixed" denotes there is a combination of self-repelling and self-attracting condensates. In the case of two condensates "mixed" means one of each kind.

Numerically, stability for trivial-phase solutions is found only for solutions satisfying the first assertion of Theorem 1. Although stability or instability is independent of the elliptic modulus $k$, the onset of instabilities is found to be accelerated by high values of $k>0.9$. 
Figure 2 shows the dynamics for a type A repulsive dn-dn solution with $V_{01}=V_{02}=-1$. Figure 3 shows a type B repulsive dn-dn solution. Both exact solutions satisfy the first part of Theorem 1. The numerical results confirm the analytical proof of stability. Note that in the numerical experiments for figures 2 and 3 both condensates are localized in the troughs of the potential. Thus the stability of these exact solutions appears similar to the stable behavior in a linear system.
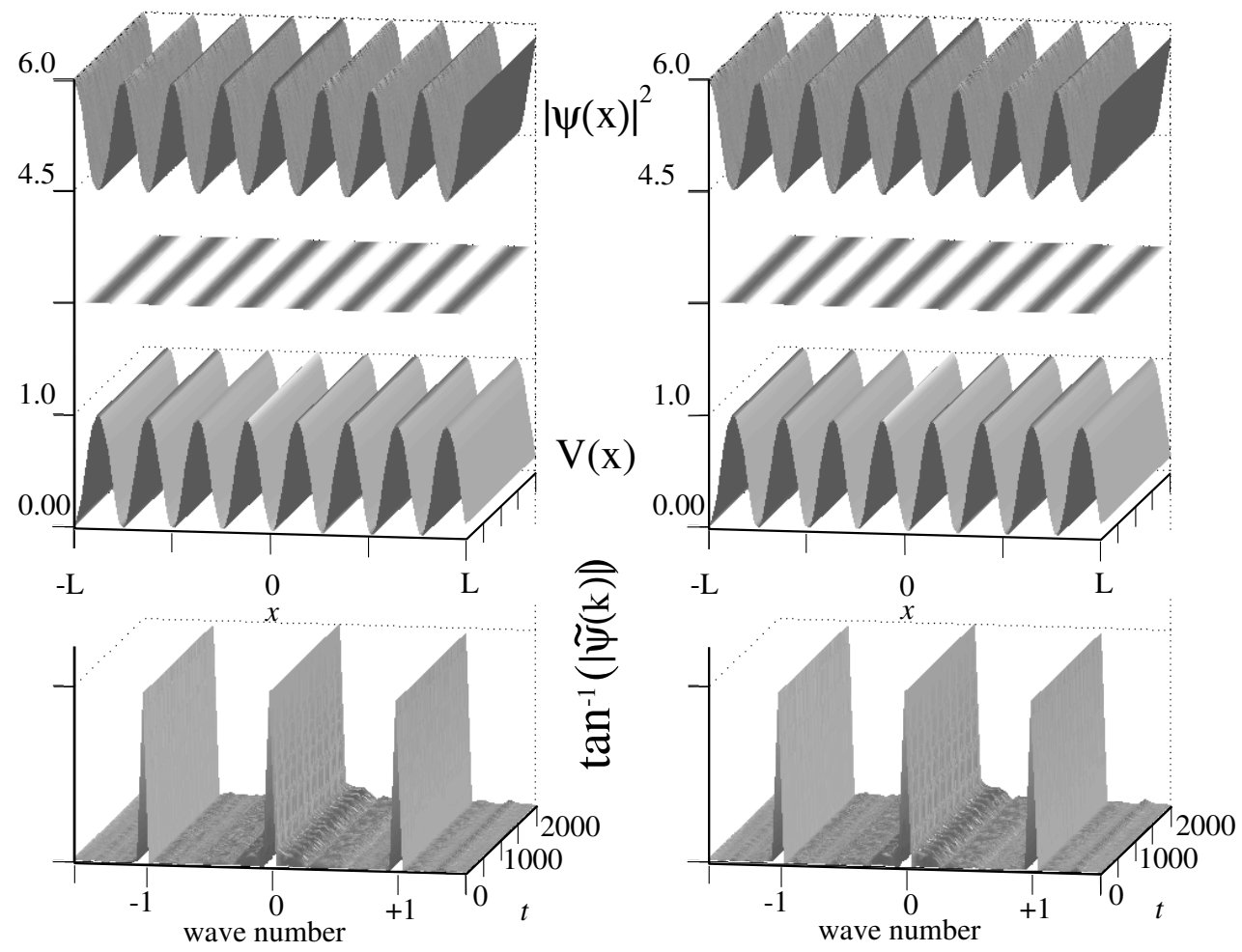

Figure 2: A stable, repulsive, type A dn-dn solution. Here $V_{01}=V_{02}=-1, \alpha=1 / 2, k=1 / 2$, and $m=1$.

Figure 4 illustrates that not all stationary repulsive dn-dn solutions are stable. The density of the wave function describing the condensate on the right is never zero, but the density of the wave function describing the condensate on the left has isolated zeros, and the first criterion of Theorem 1 no longer applies. This solution remains coherent for almost 500 time units. It displays behavior quite typical of unstable repulsive solutions. These solutions break up, and their density maxima seem drawn to the peaks of the potential. In this case, the initial condition for the condensate on the left is localized on the peaks of the potential, whereas the condensate on the right is localized in the troughs of the potential.

Figure 5 shows the typical dynamics of an attractive solution. The instability mechanisms 

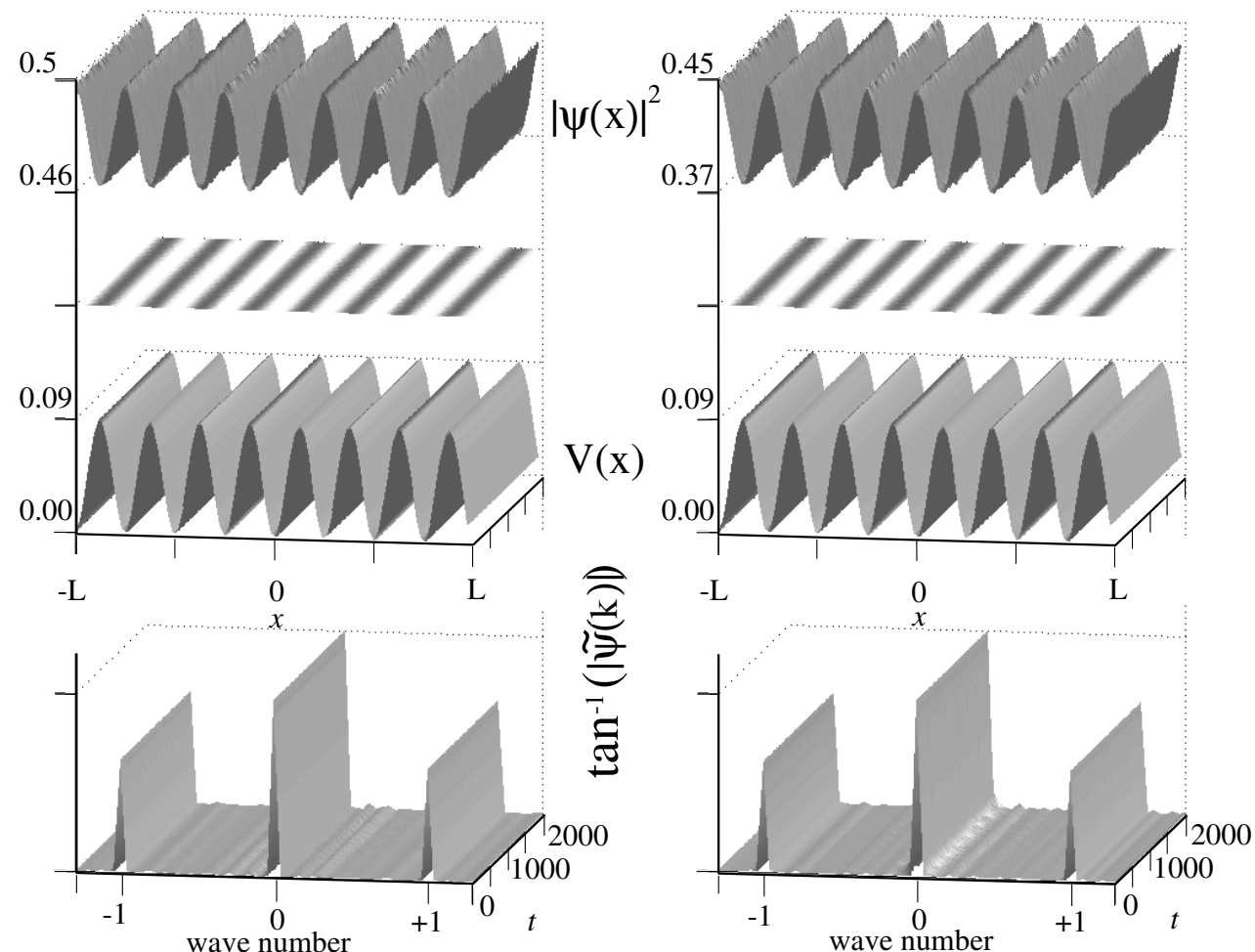

Figure 3: A stable, repulsive, type $\mathrm{B}_{3}$ dn-dn solution. Here $\alpha=1 / 2, k=1 / 2$, and $m=1$. The condensate on the left is in a $\mathrm{B}_{3 a}$ state, and the condensate on the right is in a $\mathrm{B}_{3 c}$ state.

for attractive condensates tend to focus the condensates into non-stationary peaks. Note that the instability does not occur until after approximately 300 time units, hence the configuration illustrated in figure 5 may be long-lived enough to be realized experimentally. The analogous solution for one condensate $(n=1)$ was found to be stable numerically in [20].

Figure 6 shows the dynamics of a stationary mixed dn-dn solution. Again, this solution persists for approximately 300 time units before the onset of instability. Mixed solutions also were found that persisted for more than 3000 time units. This figure allows us to analyze the process that leads to the onset of instability. The Fourier spectrum displays new modes that are gradually being activated. These new modes quickly reach amplitudes comparable to those of the original modes. This process is repeated as more modes are added, resulting in a quick evolution of the spectrum to one such as seen in figure 5.

In figure 7 the dynamics of a stable three-species $(n=3)$ repulsive dn-dn-dn condensate is shown, for the case of a positive definite interaction matrix $\boldsymbol{\alpha}$. Figure 8 displays the dynamics of an unstable type A three-species repulsive $d n-d n-d n$ condensate. Unlike the stable run of figure 7 , 

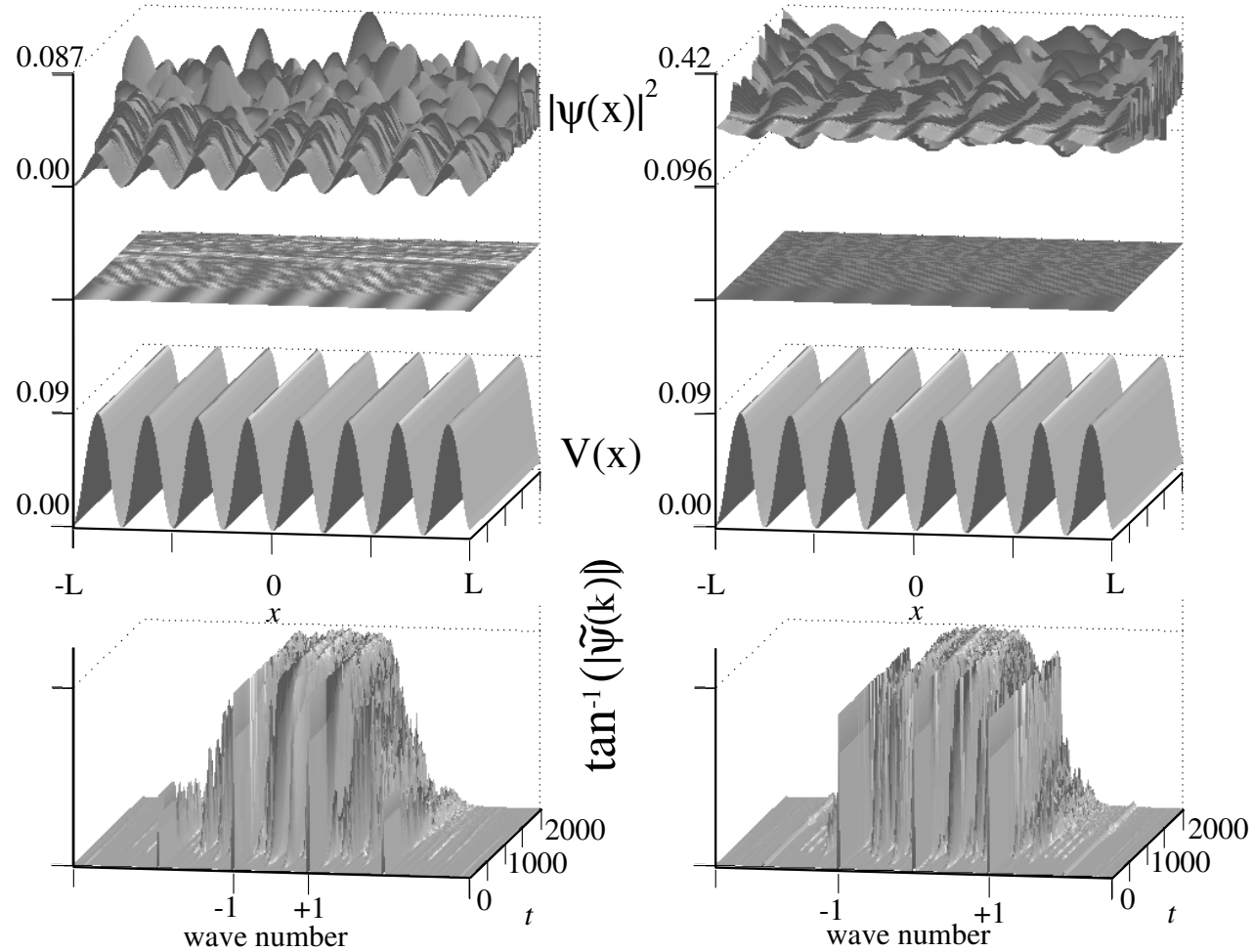

Figure 4: An unstable, repulsive, type $\mathrm{B}_{3}$ dn-dn solution. Here $\alpha=-0.4, k=1 / 2$, and $m=1$. The condensate on the left is in a $\mathrm{B}_{3 b}$ state, and the condensate on the right is in a $\mathrm{B}_{3 c}$ state.

now the interaction matrix $\boldsymbol{\alpha}$ is not positive definite and the results of Theorem 1 do not apply.

For nontrivial-phase solutions, analytical methods provide no answers. The numerics can be used to investigate the stability of these solutions. For the elliptic solutions, this is problematic, because it requires the periods of the phase and amplitude to be commensurate, effectively quantizing the parameter space of the solutions. If this is not done, the solutions are quasiperiodic. Using spectral methods to solve the initial-value problem for these solutions results in boundary effects. On the other hand, the quantization condition on parameter space is a nonlocal condition, whose solution is quite involved. In the trigonometric limit $(k \rightarrow 0)$, the phase quantization condition is automatically satisfied. It is this limit which we examine numerically.

Theorem 1 suggests that solutions whose density is never zero have the possibility of being stable for condensates with repulsive self-interaction, as long as the interaction matrix $\boldsymbol{\alpha}$ is positive definite. Indeed, the only trigonometric nontrivial phase solutions that appear to be stable numerically occur in this regime. As in [19], a sufficient amount of offset appears to be a requirement for stability. Figure 9 displays an unstable trigonometric nontrivial-phase solution, corresponding to 

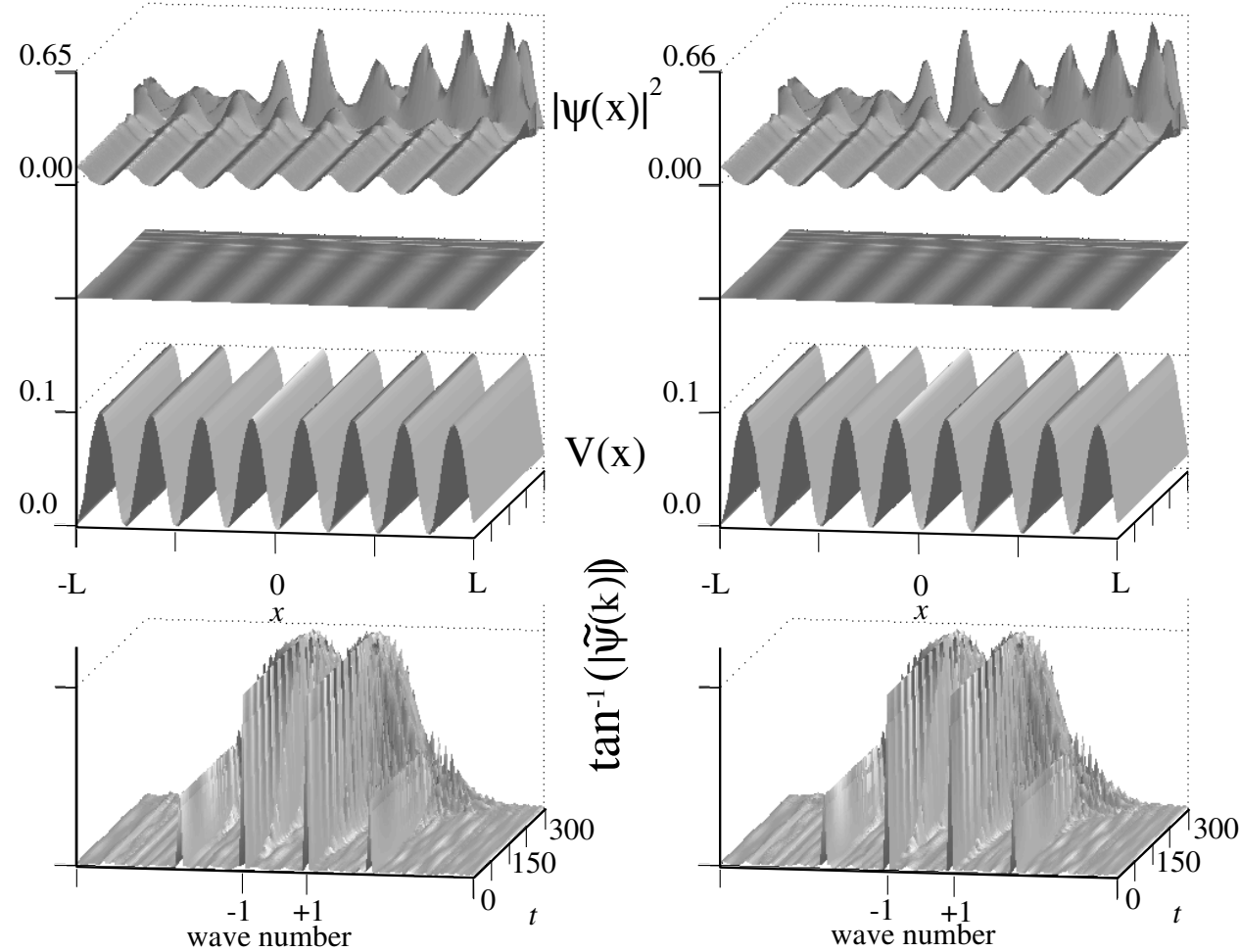

Figure 5: An unstable, attractive, type A Cn-Cn solution. Here $V_{01}=-0.1, V_{02}=-0.01, \alpha=1 / 2$, $k=\frac{1}{2}$, and $m=1$.

offset parameters $B_{1}=B_{2}=1 / 2$. On the other hand, for more offset $\left(B_{1}=B_{2}=1\right)$, it appears that the condensates are stable. Of course, the numerics do not allow such a conclusion. It does however suggest that the onset of instability time of this solution is larger than $t=2000$, the duration of the numerical runs. It is therefore conceivable that these solutions are observable, since their lifetime exceeds the duration of current experiments (see [35], for the time scale comparisons).

\section{Conclusions}

We have constructed large families of stationary solutions of the vector NLS 2 with periodic potential, modeling the interaction of different components of a Bose-Einstein Condensate in a lattice potential. Some of these solutions (Type A) degenerate to solutions of the free NLS equation as the potential strength $\rightarrow 0$. These solutions have both trigonometric and hyperbolic (soliton) limits. The trigonometric limit is especially relevant, as it models the dynamics of a multicomponent 

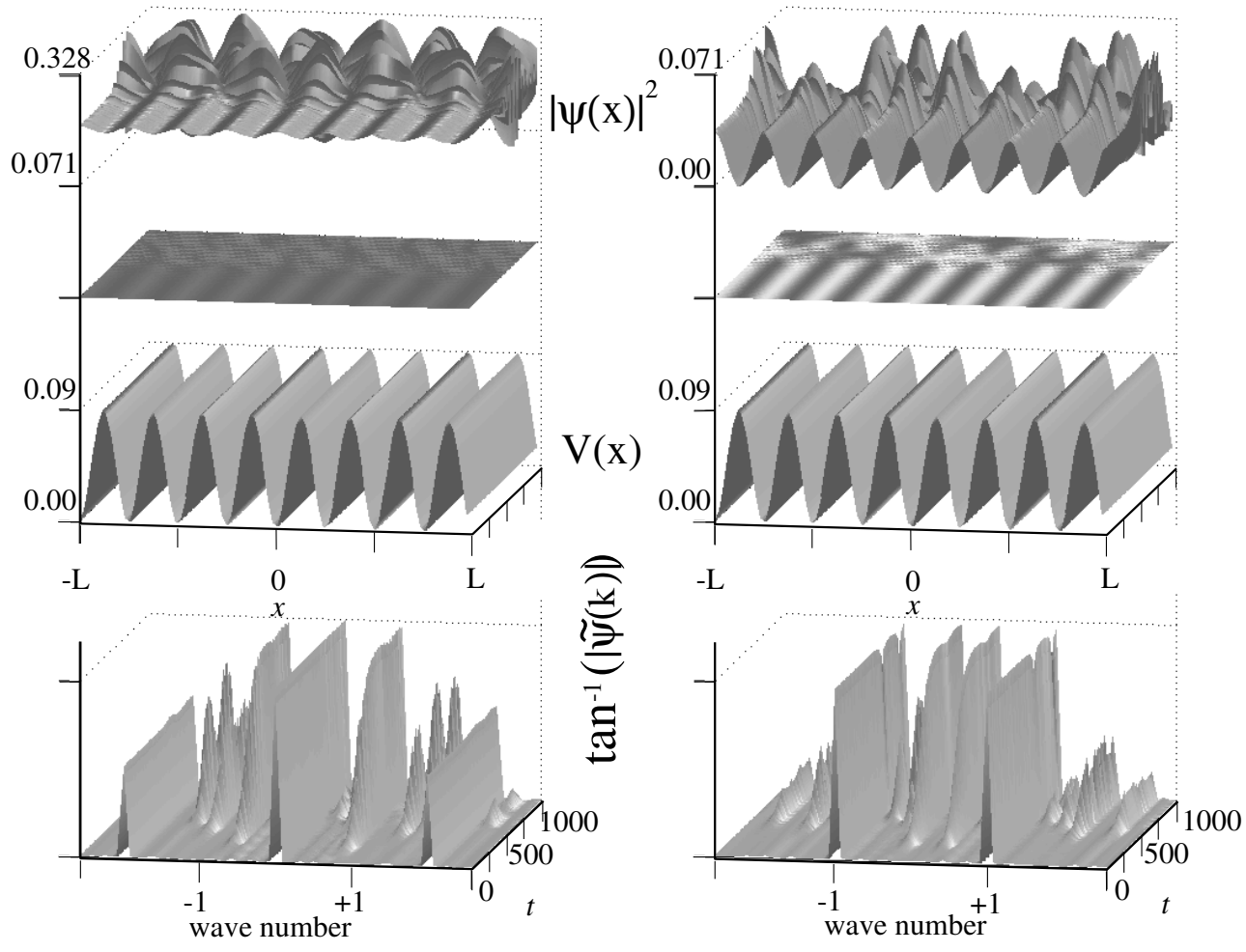

Figure 6: An unstable, type B, mixed dn-dn solution. Here $\alpha=0.4, k=1 / 2$, and $m=1$. The wave function for the condensate on the left is given by a repulsive type $\mathrm{B}_{3 c}$. The right condensate corresponds to an attractive type $\mathrm{B}_{3 d}$ wave function.

condensate in an optical potential. Other solutions (Type B) degenerate to the zero solution of the free NLS equation as the potential strength $\rightarrow 0$. These solutions have hyperbolic (soliton) limits, but no trigonometric limits. Both families of solutions have trivial-phase solutions and solutions with a nontrivial-phase profile.

For some trivial-phase solutions, stability analysis is possible: we proved that for a positivedefinite interaction matrix $\boldsymbol{\alpha}$ (mostly repulsive interactions), solutions without zeros are linearly stable. Likewise, if the interaction matrix $\boldsymbol{\alpha}$ is negative definite (mostly attractive interactions), solutions without zeros are unstable.

For all other solutions, numerical methods provide the main means of investigating their dynamical stability. Of all trivial-phase solutions, only the ones without zeros and a positive definite interaction matrix $\boldsymbol{\alpha}$ are found to be stable. For the nontrivial-phase solutions, we restricted ourselves to the trigonometric limit, in which no quantization of parameter space is required. It appears that solutions whose density profile has sufficient offset are stable, or at least that their onset of 

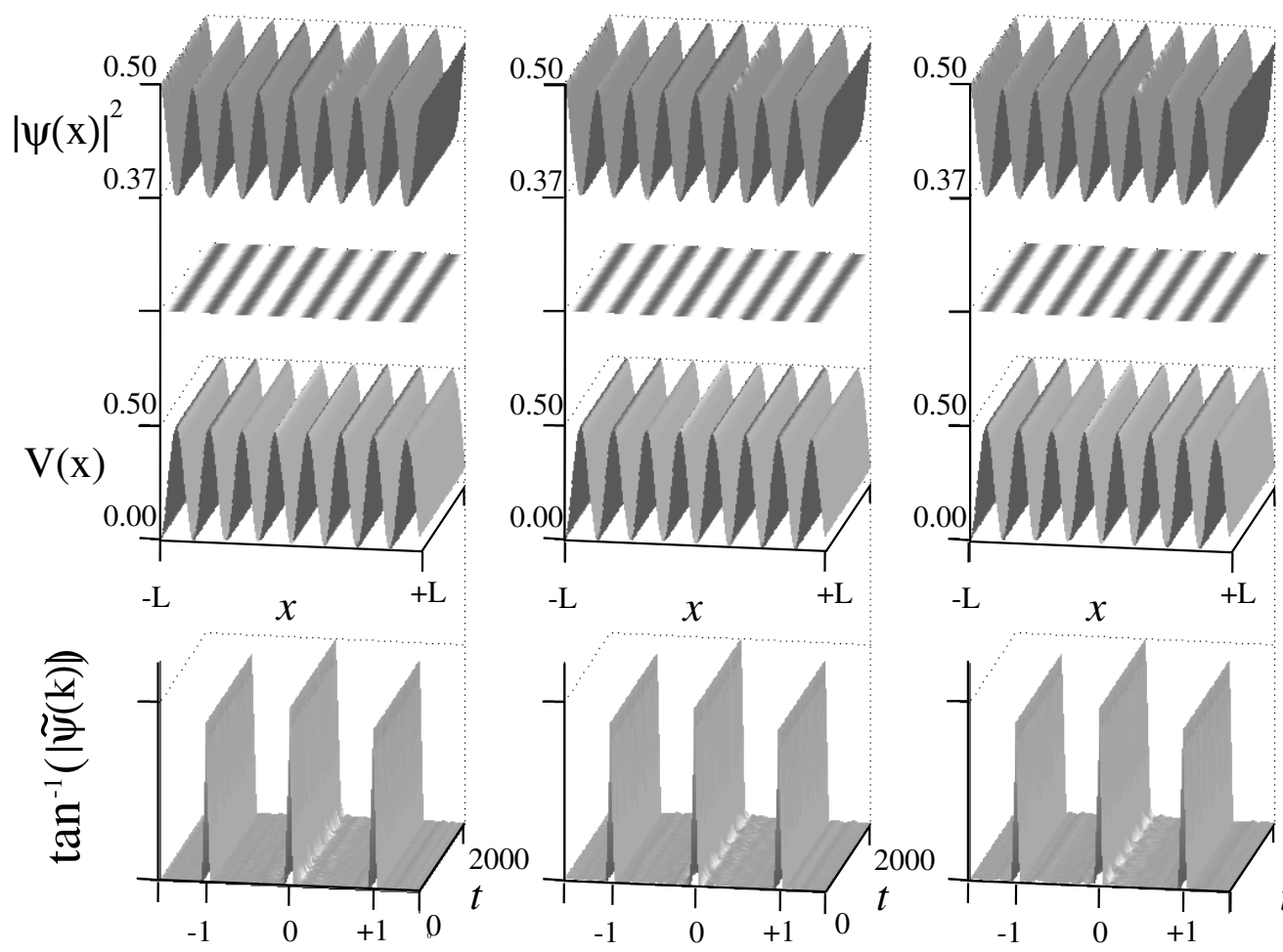

wave number

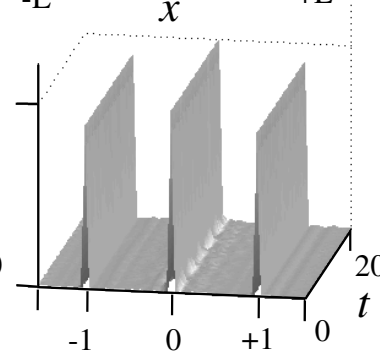

wave number

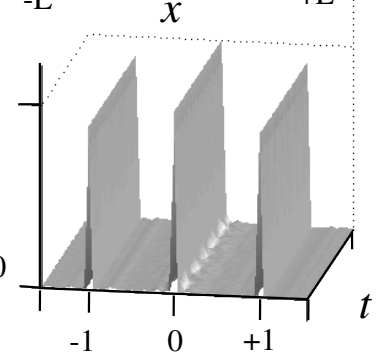

wave number

Figure 7: A stable, 3-species, repulsive dn-dn-dn solution. Here $V_{01}=V_{02}=V_{03}=-1 / 2, k=1 / 2$, $m=1$, and the interaction matrix $\boldsymbol{\alpha}$ is positive definite.

instability time becomes unbounded as the offset becomes infinite.

\section{Acknowledgements}

This work was supported by NSF grants DMS-0071568 (B.D.), DMS-0092682 (J.N.K.) and DMS9810726. (VIGRE) (M.S.P. and B. W. W.)

\section{References}

[1] N. H. Anderson, J. R. Ensher, M. R. Matthews, C. E. Wieman and E. A. Cornell, Science 269, 198 (1995).

[2] W. Ketterle, D. S. Sturfee, and D. M. Stamper-Kurn, in Proceedings of the International School of Physics "Enrico Fermi" (IOS Press, Amsterdam; Washington, D.C., 1999), pp. 67-176. 

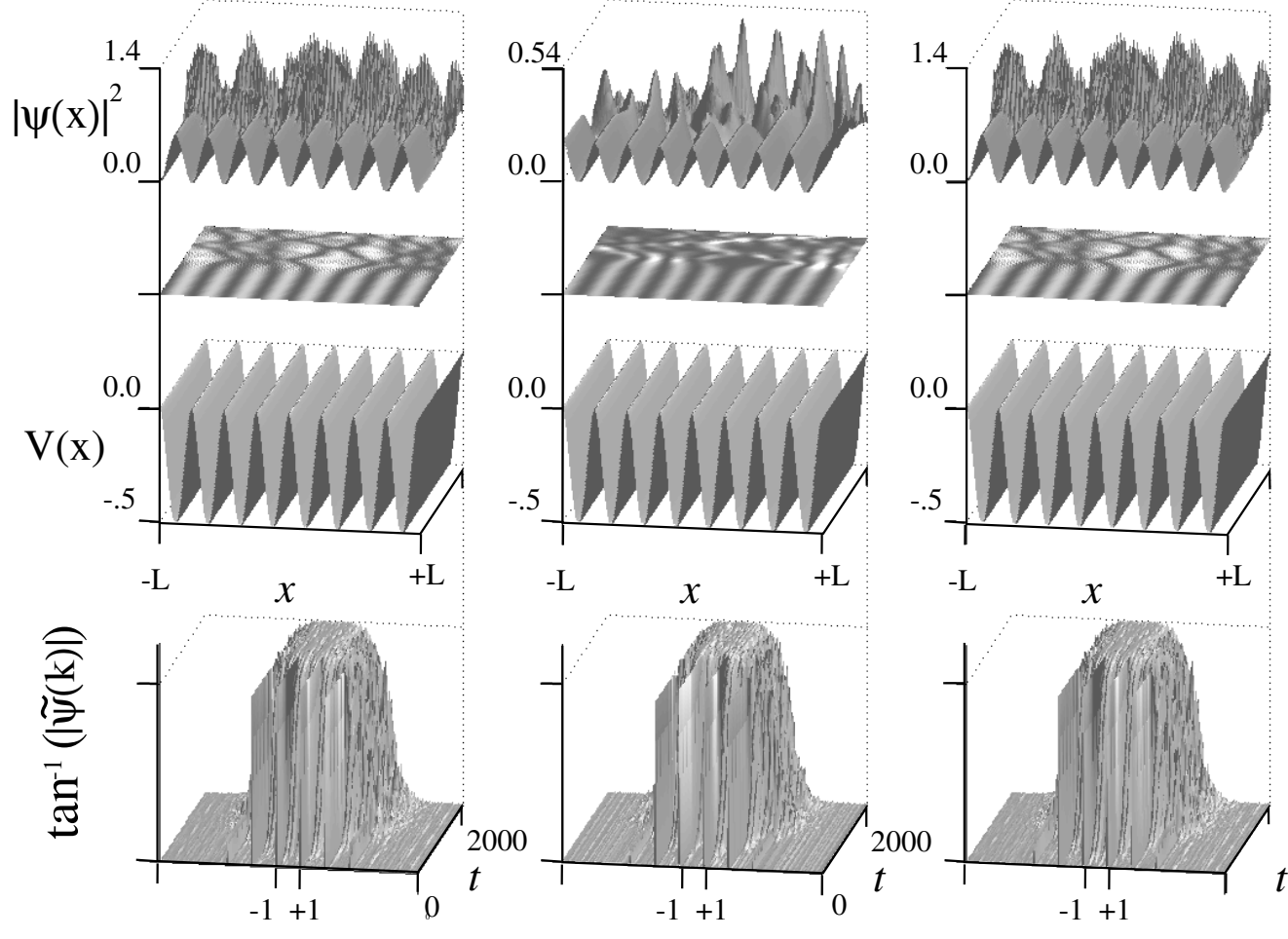

wave number

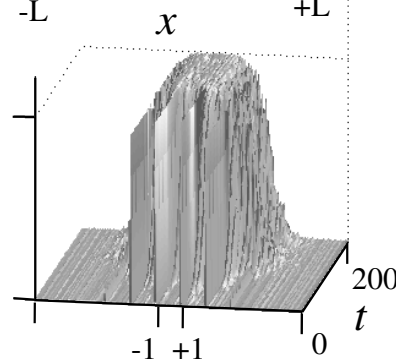

wave number

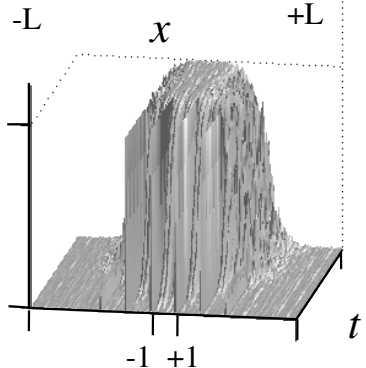

wave number

Figure 8: An unstable, 3-species, repulsive dn-dn-dn solution with $V_{01}=V_{02}=V_{03}=\frac{1}{2}, k=\frac{1}{2}$, $m=1$, and the interaction matrix $\boldsymbol{\alpha}$ that is not positive definite.

[3] F. Dalfovo, S. Giorgini, L. P. Pitaevskii, and S. Stringari, Rev. Mod. Phys. 71, 463 (1999).

[4] T. Tsurumi, H. Morise, and M. Wadati, Int. J. Mod. Phys. 14655 (2000)

[5] K. Huang, Statistical Mechanics, (John Wiley, New York, 1963).

[6] K. Bongs, S. Burger, S. Dettmer, D. Hellweg, J. Arlt, W. Ertmer, and K. Sengstock, Phys. Rev. A 63, 031602(R) (2001).

[7] B. P. Anderson and M. A. Kasevich, Science 282, 1686 (1998).

[8] E. W. Hagley, L. Deng, L. Kozuma, J. Wen, K. Helmerson, S. L. Rolston, W. D. Phillips, Science 283, 1706 (1999).

[9] M.L. Chiofalo and M.P. Tosi, Phys. Lett. A 268406 (2000). 

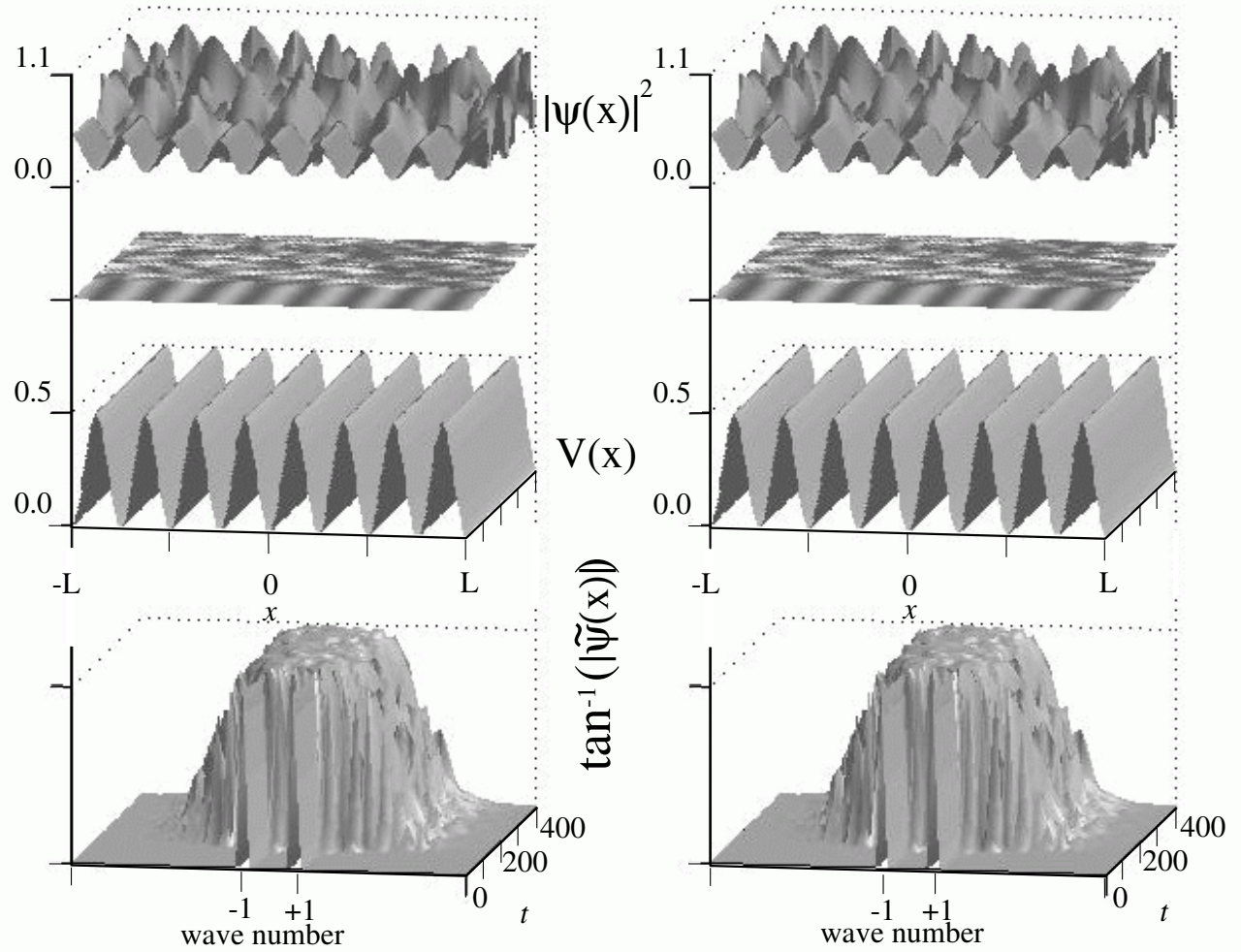

Figure 9: An unstable, 2-species, repulsive trigonometric nontrivial phase solution with $V_{01}=V_{02}=$ $-\frac{1}{2}, \alpha=1 / 2, B_{1}=B_{2}=1 / 2$, and $m=1$.

[10] Y. B. Ovchinnikov, J. H. Müller, M. R. Doery, E. J. D. Vredenbregt, K. Helmerson, S. L. Rolston, W. D. Phillips, Phys. Rev. Lett. 83, 284 (1999).

[11] D.-I. Choi and Q. Niu, Phys. Rev. Lett. 82, 2022 (1999).

[12] D. Jaksch, C. Bruder, J. I. Cirac, C. W. Gardiner, and P. Zoller, Phys. Rev. Lett. 81, 3108 (1998);

[13] G. K. Brennen, C. M. Caves, P. S. Jessen, and I. H. Deutsch, Phys. Rev. Letts. 82, 1060 (1999).

[14] C. J. Myatt, E. A. Burt, R. W. Ghrist, E. A. Cornell, and C. E. Wieman, Phys. Rev. Lett. 78, 586 (1997).

[15] D. S. Hall, M. R. Matthews, J. R. Ensher, C. E. Wiemann, and E. A. Cornell, Phys. Rev. Lett. 81, 1539 (1998). 

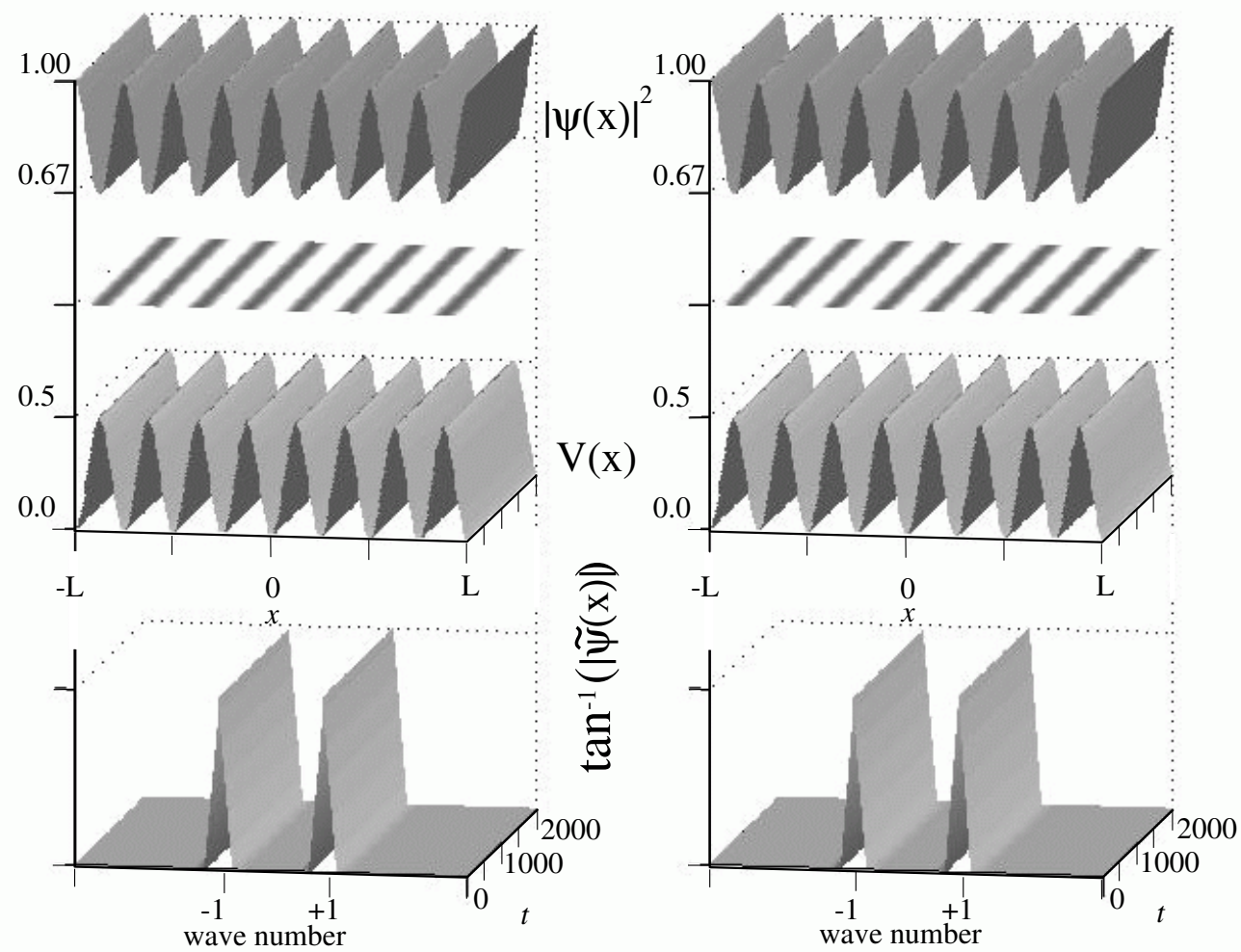

Figure 10: A numerically stable, 2-species, repulsive trigonometric nontrivial phase solution with $V_{01}=V_{02}=-\frac{1}{2}, \alpha=1 / 2, B_{1}=B_{2}=1$, and $m=1$.

[16] D. M. Stamper-Kurn, M. R. Andrews, A. P. Chikkatur, S. Inouye, H.-J. Miesner, J. Stenger, and W. Ketterle, Phys. Rev. Lett. 80, 2027 (1998).

[17] G. Modugno, M. Modugno, F. Riboli, G. Roati, and M. Inguscio, Phys. Rev. Lett. 89, 190404 (2002).

[18] J. C. Bronski, L. Carr, B. Deconinck, and J. N. Kutz, Phys. Rev. Lett. 86, 1402 (2001).

[19] J. C. Bronski, L. Carr, B. Deconinck, J. N. Kutz, and K. Promislow, Phys. Rev. E 63, 036612 (2001).

[20] J. C. Bronski, L. Carr, R. Carretero-Gonzalez, B. Deconinck, J. N. Kutz, and K. Promislow, Phys. Rev. E 64, 056615 (2001).

[21] G. Baym, Lectures in Quantum Mechanics, (Addison-Wesley, Redwood City, CA), Ch. 20. 
[22] L. P. Pitaevskii, Sov. Phys. JETP 13, 451 (1961).

[23] E. P. Gross, Nuovo Cimento 20, 454 (1961).

[24] E. H. Lieb, R. Seiringer, and J. Yngvason, Phys. Rev. A 61, 043602 (2000)

[25] E. H. Lieb and R. Seiringer, Phys. Rev. Lett. 88, 170409 (2002).

[26] D. S. Petrov, M. Holzmann, and G. V. Shylapnikov, Phys. Rev. Letts. 84, 2551 (2000).

[27] D.S. Petrov, G.V. Shlyapnikov, and J.T.M. Walraven, Phys. Rev. Lett. 85, 3745 (2000).

[28] L.D. Carr, C.W. Clark, and W.P. Reinhardt, Phys. Rev. A 62063610 and 063611 (2000).

[29] L.D. Carr, M. A. Leung, and W.P. Reinhardt, J. Phys. B 33, 3983 (2000).

[30] B. D. Esry, C. H. Greene, J. P. Burke, Jr., and J. L. Bohn, Phys. Rev. Lett. 78, 0013-9007 (1997)

[31] Th. Busch, J. I. Cirac, V. M. Pérez-García, and P. Zoller, Phys. Rev. A 56, 1050-2947 (1997)

[32] M. Abramowitz and I. Stegun (eds), Handbook of mathematical functions (National Bureau of Standards, Applied Mathematics Series, 55, 1964)

[33] Matrix Theory. Vol. 1, F. R. Gantmacher, (AMS-Chelsea, 2000).

[34] R. Courant and D. Hilbert, Methods of Mathematical Physics, (Wiley, New York, 1989).

[35] B. Deconinck, B. A. Frigyik, and J. N. Kutz, J. Nonlinear Sci. 12, 169-205 (2002) 